\title{
An engineered mayhem: YAP/TAZ mechanosignaling and hepatocarcinogenesis in NAFLD
}

\author{
Gyorgy Baffy ${ }^{1,2} *$ (1) \\ ${ }^{1}$ VA Boston Healthcare System, Boston, Massachusetts 02130, USA \\ ${ }^{2}$ Harvard Medical School, Boston, Massachusetts 02115, USA
}

*Correspondence: Gyorgy Baffy, VA Boston Healthcare System, 150 S, Huntington Avenue, Boston, Massachusetts 02130, USA. gbaffy@bwh.harvard.edu

Academic Editor: Amedeo Lonardo, Azienda Ospedaliero-Universitaria di Modena, Italy

Received: May 26, 2021 Accepted: June 17, 2021 Published: August 31, 2021

Cite this article: Baffy G. An engineered mayhem: YAP/TAZ mechanosignaling and hepatocarcinogenesis in NAFLD. Explor Med. 2021;2:305-10. https://doi.org/10.37349/emed.2021.00048

\section{Keywords}

Mechanocrine signaling, mechanotransduction, hepatocellular carcinoma, Hippo signaling, liver regeneration

Nonalcoholic fatty liver disease (NAFLD) is estimated to affect two billion individuals worldwide, including $25-30 \%$ of the US population [1]. NAFLD has a continuous spectrum from steatosis to steatohepatitis with a variable degree of liver fibrosis, associated with an increased risk of progression into cirrhosis [2]. In addition, a growing proportion of hepatocellular carcinoma (HCC) has been attributed to NAFLD in the past decades [3]. NAFLD-associated HCC is mostly diagnosed in the setting of cirrhosis, but HCC also complicates NAFLD in the absence of significant fibrosis [3]. The incidence of NAFLD-associated HCC in non-cirrhotic livers is generally less than 1 per 1000 patient-years, making cancer surveillance impractical in the absence of cirrhosis [4]. However, the population-attributable fraction of non-cirrhotic HCC is substantial due to the high overall prevalence of NAFLD [5]. To develop efficient biomarkers and novel targets for the prevention and treatment of NAFLD-associated HCC, we need therefore an improved understanding of the cellular and molecular mechanisms of hepatocarcinogenesis.

In a recent work, Hyun etal. [6] investigated the potential role of theyes-associated protein/transcriptional coactivator with PDZ-binding motif (YAP/TAZ) pathway in NAFLD-associated liver tumorigenesis. YAP, a member of the Src protein kinase family and its paralog, the TAZ, have a complex role in liver pathobiology including the development of liver cancer [7]. In resting cells, YAP is marked for cytoplasmic sequestration and degradation by upstream kinases, while dephosphorylated YAP becomes active and translocates to the nucleus where it promotes the transcription of genes that regulate cell growth and proliferation [8]. YAP/ TAZ have been shown to induce de-differentiation of various cell types and sustained activation of YAP/TAZ is now recognized as a key mechanism of oncogenesis [8].

YAP/TAZ activity is regulated by neurofibromatosis 2 (NF2) or merlin, a tumor suppressor protein identified in the context of neurofibromatosis [9]. NF2 acts as a scaffold protein modulating several signaling pathways of cell proliferation and survival. Also, dephosphorylated YAP/TAZ may induce NF2, creating 
a negative feedback loop that terminates further YAP/TAZ activation [10]. NF2 activity is controlled in hepatocytes by the epithelial splicing regulatory protein-2 (ESRP2), a cell-specific RNA splicing factor, which generates an adult NF2 transcript that is more efficient than its fetal variant [11].

With this background knowledge, Hyun et al. [6] set out to analyze changes in the ESRP2-NF2-YAP/ TAZ pathway in response to experimental steatohepatitis induced by choline-deficient, L-amino acid defined high-fat diet (CDAHFD) in mice. Their aim was to find out if YAP/TAZ is dysregulated in steatohepatitis in a way that would link the pathway to increased risk of HCC and/or cholangiocarcinoma in their model. They found that expression and nuclear localization of ESRP2 was inversely associated with tumor necrosis factor-alpha expression and with histological features of inflammation and hepatocellular injury in CDAHFD mice. These changes paralleled other findings such as higher proportions of the fetal NF2 variant, YAP dephosphorylation, TAZ accumulation and the expression of multiple YAP/TAZ target genes, indicating the presence of upregulated pathways for cell proliferation and survival. Moreover, changes in NF2 splice variants correlated with histological improvement when experimental NAFLD was reversed [6].

Hyun et al. [6] extrapolated their findings to human NAFLD by utilizing publicly accessible liver RNA databases and an unrelated tissue bank for further analysis, which indicated that NAFLD livers exhibit significantly reduced levels of both ESRP2 and adult NF2, while HCC samples showed particular abundance of fetal NF2. To establish a functional relationship between ESRP2 expression, NF2 messenger RNA splicing, and YAP/TAZ activation in liver tumorigenesis, Hyun et al. [6] overexpressed ESRP2 in the human HCC-derived cell line Huh-7, which resulted in reduced levels of fetal NF2 and less activation of YAP/TAZ. In addition, the authors used clustered regularly interspaced short palindromic repeats (CRISPR) to enrich Huh-7 cells with fetal NF2, which caused increased YAP/TAZ activity and target gene expression. Finally, The Cancer Genome Atlas (TCGA) database analysis demonstrated that higher HCC recurrence and reduced overall survival and progression-free survival were significantly more likely in tumors with lower ESRP2 expression and enriched with the mRNA encoding fetal NF2 [6].

The work of Hyun et al. [6] identifies a novel molecular mechanism of liver tumorigenesis in NAFLD. The authors concluded that inflammation has a key role in ESRP2-mediated disruption of YAP/TAZ signaling in their model of experimental NAFLD [6]. Since the risk of cholangiocarcinoma is also increased in NAFLD [12], yet bile duct cells are not directly affected by ectopic lipid accumulation, the authors argued that altered metabolic pathways are therefore less likely to account for these changes. Association between ESRP2 splicing variants and fibrosis was not addressed in this work, but an earlier report from the same group described YAP accumulation in ductular liver cells with progenitor capabilities corresponding with the degree of fibrosis in human and experimentally induced NAFLD [13]. It is important to recall, however, that NAFLD-associated HCC has been reported in patients without histological evidence of steatohepatitis [14], supporting the notion that an oncogenic milieu may already exist in early NAFLD in the absence of inflammation. In fact, presence of large lipid droplets correlated with increased nuclear translocation of YAP in fatty acid-loaded primary human hepatocytes, suggesting that nuclear displacement alone may be a sufficient mechanical stimulus to activate the pathway [15].

YAP/TAZ has multiple upstream regulators with the Hippo signaling pathway being the foremost, albeit not exclusive, tumor suppressor apparatus controlling its activity [8]. Hippo was originally described in Drosophila where its inactivation resulted in considerable overgrowth (hence the name) due to excessive cellular proliferation and reduced rates of apoptosis [16]. The Hippo signaling pathway [including the human kinase homologs mammalian Ste20-like kinase (MST) and large tumor suppressor kinase (LATS)] was subsequently identified as a major regulator of organ development and regeneration [7, 17]. Partial hepatectomy promptly induces YAP/TAZ activation, corresponding to forceful liver cell proliferation and growth before it becomes inactive again as the original tissue mass is quickly regained [18]. This aspect of liver regeneration, marveled for precise termination as much as for initiation, has been termed 'hepatostat' [19]. It is now clear that mechanical cues from cell density, polarity and other structural properties of the cellular microenvironment are critically important in Hippo-dependent and Hippo-independent regulation of 
YAP/TAZ activation, and this response may be modulated by inflammatory and metabolic changes in the regenerating liver $[7,8]$.

Mechanotransduction is the umbrella term for cellular responses to physical stimuli detected by mechanosensors and transmitted into biochemical signals [20]. YAP/TAZ have a fundamental role in mechanocrine signaling pathways, governing the interaction of various liver cells with each other and with the extracellular matrix (ECM) [18]. Abnormal levels of shear, stretch and compression forces stimulate defenestration and capillarization of liver sinusoidal endothelial cells (LSECs), worsen lipid accumulation in hepatocytes, and allows the transformation of hepatic stellate cells (HSCs) to myofibroblasts with increased rates of ECM production (Figure 1). These histological hallmarks become more prominent with the progression of NAFLD, leading to tissue stiffness, disruption of cell-cell adhesions, and sustained activation of key mechanosensors such as integrins and focal adhesion kinase (FAK), involving the cytoskeleton, intracellular cascades (mitogen-activated protein kinases and Rho kinases) and signaling radicals (calcium, nitric oxide and reactive oxygen intermediates), engaging YAP/TAZ and promoting cell growth and proliferation which may ultimately lead to liver cancer [7,21]. This undesirable outcome is less probable in early stages of NAFLD in the absence of significant fibrosis, but-as alluded to this above-we have now ample evidence that it happens more often than initially thought.
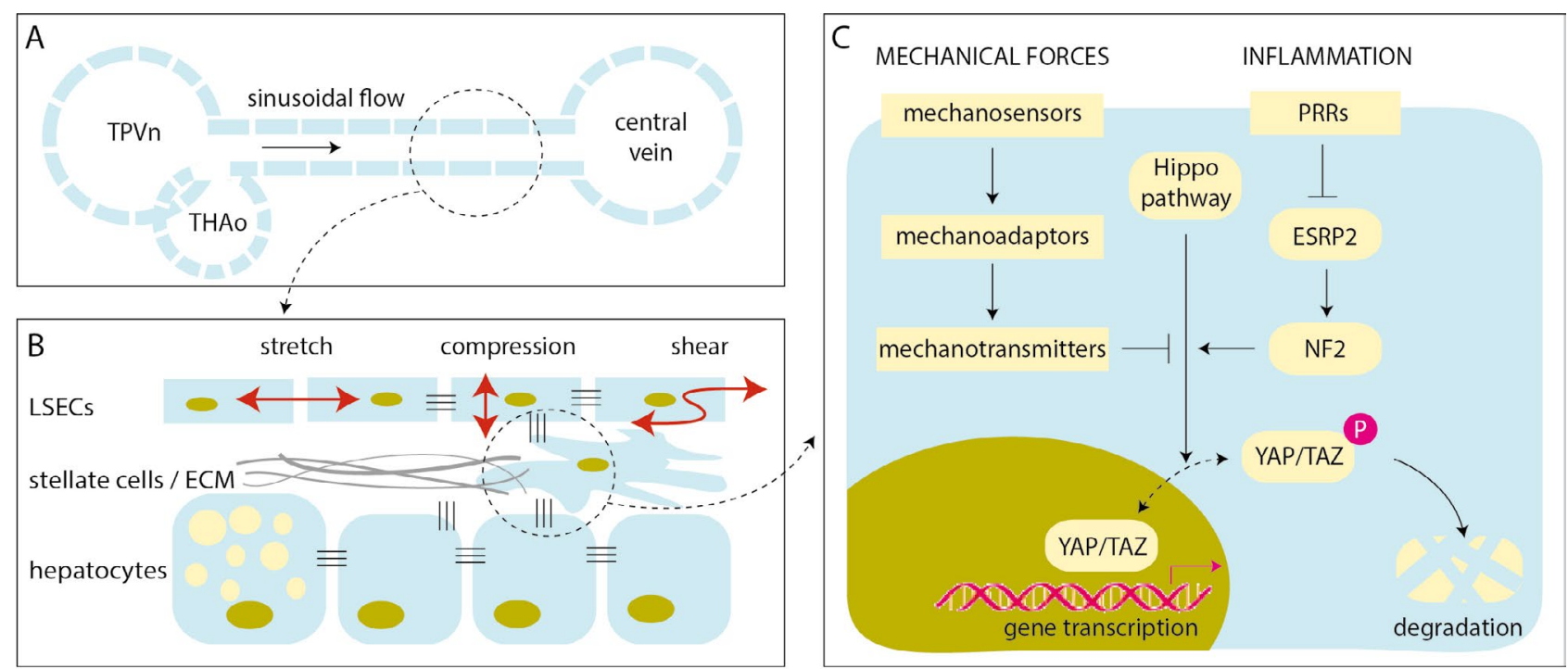

Figure 1. Mechanotransduction and the regulation of YAP/TAZ in NAFLD. A. Schematic illustration of a liver sinusoid with blood flowing from the confluence of terminal portal venule (TPVn) and terminal hepatic arteriole (THAo) toward the central vein; B. LSECs are subjected to various forms of mechanical forces by the blood flow (red arrows). LSECs may respond with endothelial dysfunction, causing defenestration and capillarization, worsening hepatocellular steatosis, activating stellate cells that may contract and produce ECM, initiating angiogenesis, microthrombosis, and elevating the portal pressure (many of these are not shown in this scheme). These events stiffen the liver and activate mechanotransduction pathways with multiple amplification loops; C. schematic diagram of the YAP/TAZ pathway in a liver cell. Mechanical forces and inflammation may act synergistically and weaken Hippo-mediated phosphorylation and degradation of YAP/TAZ, which is then translocated to the nucleus and promotes cell growth and proliferation. P: phosphate; PRR: patter recognition receptor

How is YAP/TAZ mechanosignaling different during a powerful yet carefully orchestrated response in liver regeneration from when it becomes a sustained oncogenic force? While liver regeneration may or may not be significantly affected by steatosis as demonstrated in living-related liver transplantation [22], the work of Hyun et al. [6] suggests that concomitant inflammation (and plausibly fibrosis) is more likely to interfere with YAP/TAZ and its upstream regulation. Early events of mechanotransduction in NAFLD still remain incompletely understood. Pathologists warn us that some degree of lobular or portal inflammation is present in most NAFLD cases suggesting that 'pure' steatosis may not really exist [23]. Moreover, early work proposed that sudden increase in portal pressure following partial hepatectomy may account for shear stress in the liver remnant [24], tempting us to speculate that elevated portal pressure-even before significant fibrosis develops-could be a factor in initiating YAP/TAZ-associated mechanotransduction and contributing to development of HCC in early-stage NAFLD. Nuclear translocation of YAP/TAZ has been shown to predict 
poor survival of HCC patients [25], but how this molecular event could be utilized as an early biomarker of hepatocarcinogenesis will require further studies. Furthermore, YAP/TAZ activation as a pharmaceutical target may prove to be challenging as it has pleiotropic effects shared by multiple upstream molecular cascades [26]. For now, our best strategy in NAFLD management is to prevent steatohepatitis and enhance disease monitoring in its presence. At least until we find a safe key to fine tune the YAP/TAZ machinery of mechanotransduction.

\section{Abbreviations}

ECM: extracellular matrix

ESRP2: epithelial splicing regulatory protein-2

HCC: hepatocellular carcinoma

LSECs: liver sinusoidal endothelial cells

NAFLD: nonalcoholic fatty liver disease

NF2: neurofibromatosis 2

TAZ: transcriptional coactivator with PDZ-binding motif

YAP: yes-associated protein

\section{Declarations}

Author contributions

The author contributed solely to the work.

\section{Conflicts of interest}

The author declares that he has no conflicts of interest.

\section{Ethical approval}

Not applicable.

\section{Consent to participate}

Not applicable.

\section{Consent to publication}

Not applicable.

Availability of data and materials

Not applicable.

\section{Funding}

Not applicable.

\section{Copyright}

(C) The Author(s) 2021.

\section{References}

1. Younossi ZM. Non-alcoholic fatty liver disease - a global public health perspective. J Hepatol. 2019;70:531-44.

2. Powell EE, Wong VW, Rinella M. Non-alcoholic fatty liver disease. Lancet. 2021;397:2212-24.

3. Huang DQ, El-Serag HB, Loomba R. Global epidemiology of NAFLD-related HCC: trends, predictions, risk factors and prevention. Nat Rev Gastroenterol Hepatol. 2021;18:223-38. 
4. Kanwal F, Kramer JR, Mapakshi S, Natarajan Y, Chayanupatkul M, Richardson PA, et al. Risk of hepatocellular cancer in patients with non-alcoholic fatty liver disease. Gastroenterology. 2018; 155:1828-37.e2.

5. Baffy G. Hepatocellular carcinoma in non-alcoholic fatty liver disease: epidemiology, pathogenesis, and prevention. J Clin Transl Hepatol. 2013;1:131-7.

6. Hyun J, Al Abo M, Dutta RK, Oh SH, Xiang K, Zhou X, et al. Dysregulation of the ESRP2-NF2-YAP/TAZ axis promotes hepatobiliary carcinogenesis in non-alcoholic fatty liver disease. J Hepatol. 2021;[Epub ahead of print].

7. Patel SH, Camargo FD, Yimlamai D. Hippo signaling in the liver regulates organ size, cell fate, and carcinogenesis. Gastroenterology. 2017;152:533-45.

8. Zanconato F, Cordenonsi M, Piccolo S. YAP/TAZ at the roots of cancer. Cancer Cell. 2016;29:783-803.

9. Lutchman M, Rouleau GA. The neurofibromatosis type 2 gene product, schwannomin, suppresses growth of NIH 3T3 cells. Cancer Res. 1995;55:2270-4.

10. Moroishi T, Park HW, Qin B, Chen Q, Meng Z, Plouffe SW, et al. A YAP/TAZ-induced feedback mechanism regulates Hippo pathway homeostasis. Genes Dev. 2015;29:1271-84.

11. Bangru S, Arif W, Seimetz J, Bhate A, Chen J, Rashan EH, et al. Alternative splicing rewires Hippo signaling pathway in hepatocytes to promote liver regeneration. Nat Struct Mol Biol. 2018;25:928-39.

12. Welzel TM, Graubard BI, Zeuzem S, El-Serag HB, Davila JA, McGlynn KA. Metabolic syndrome increases the risk of primary liver cancer in the United States: a study in the SEER-Medicare database. Hepatology. 2011;54:463-71.

13. Machado MV, Michelotti GA, Pereira TA, Xie G, Premont R, Cortez-Pinto H, et al. Accumulation of duct cells with activated YAP parallels fibrosis progression in non-alcoholic fatty liver disease. J Hepatol. 2015;63:962-70.

14. Guzman G, Brunt EM, Petrovic LM, Chejfec G, Layden TJ, Cotler SJ. Does nonalcoholic fatty liver disease predispose patients to hepatocellular carcinoma in the absence of cirrhosis? Arch Pathol Lab Med. 2008;132:1761-6.

15. Chin L, Theise ND, Loneker AE, Janmey PA, Wells RG. Lipid droplets disrupt mechanosensing in human hepatocytes. Am J Physiol Gastrointest Liver Physiol. 2020;319:G11-22.

16. Huang J, Wu S, Barrera J, Matthews K, Pan D. The Hippo signaling pathway coordinately regulates cell proliferation and apoptosis by inactivating Yorkie, the Drosophila Homolog of YAP. Cell. 2005;122:421-34.

17. Meng Z, Moroishi T, Guan KL. Mechanisms of Hippo pathway regulation. Genes Dev. 2016;30:1-17.

18. Song Z, Gupta K, Ng IC, Xing J, Yang YA, Yu H. Mechanosensing in liver regeneration. Semin Cell Dev Biol. 2017;71:153-67.

19. Michalopoulos GK. Hepatostat: liver regeneration and normal liver tissue maintenance. Hepatology. 2017;65:1384-92.

20. Kang N. Mechanotransduction in liver diseases. Semin Liver Dis. 2020;40:84-90.

21. Filliol A, Schwabe RF. Contributions of fibroblasts, extracellular matrix, stiffness, and mechanosensing to hepatocarcinogenesis. Semin Liver Dis. 2019;39:315-33.

22. Wu C, Lu C, Xu C. Short-term and long-term outcomes of liver transplantation using moderately and severely steatotic donor livers: a systematic review. Medicine (Baltimore). 2018;97:e12026.

23. Brunt EM, Kleiner DE, Wilson LA, Unalp A, Behling CE, Lavine JE, et al; NASH Clinical Research Network. Portal chronic inflammation in nonalcoholic fatty liver disease (NAFLD): a histologic marker of advanced NAFLD-Clinicopathologic correlations from the nonalcoholic steatohepatitis clinical research network. Hepatology. 2009;49:809-20.

24. Sato Y, Koyama S, Tsukada K, Hatakeyama K. Acute portal hypertension reflecting shear stress as a trigger of liver regeneration following partial hepatectomy. Surg Today. 1997;27:518-26. 
25. Van Haele M, Moya IM, Karaman R, Rens G, Snoeck J, Govaere O, et al. YAP and TAZ heterogeneity in primary liver cancer: an analysis of its prognostic and diagnostic role. Int J Mol Sci. 2019;20:638.

26. Dobrokhotov O, Samsonov M, Sokabe M, Hirata H. Mechanoregulation and pathology of YAP/TAZ via Hippo and non-Hippo mechanisms. Clin Transl Med. 2018;7:23. 